\title{
Electrically conductive coatings consisting of Ag-decorated cellulose nanocrystals
}

\author{
Nicole Meulendijks • Marieke Burghoorn • Renz van Ee - Maurice Mourad - Daniel Mann • \\ Helmut Keul $\cdot$ Guy Bex $\cdot$ Emile van Veldhoven $\cdot$ Marcel Verheijen $\cdot$ Pascal Buskens (i)
}

Received: 20 January 2017/ Accepted: 23 February 2017/Published online: 2 March 2017

(C) The Author(s) 2017. This article is published with open access at Springerlink.com

\begin{abstract}
For the preparation of electrically conductive composites, various combinations of cellulose and conducting materials such as polymers, metals, metal oxides and carbon have been reported. The conductivity of these cellulose composites reported to date ranges from $10^{-6}$ to $10^{3} \mathrm{~S} \mathrm{~cm}^{-1}$. Cellulose nanocrystals (CNCs) are excellent building blocks for the production of high added value coatings. The essential process steps for preparing such coatings, i.e. surface modification of CNCs dispersed in water and/or alcohol followed by application of the dispersion to substrate samples using dip coating, are low cost and easily scalable. Here, we present coatings consisting of $\mathrm{Ag}$ modified CNCs that form a percolated network upon solvent evaporation.
\end{abstract}

Electronic supplementary material The online version of this article (doi:10.1007/s10570-017-1240-y) contains supplementary material, which is available to authorized users.

N. Meulendijks $\cdot$ M. Burghoorn · R. van Ee ·

M. Mourad · E. van Veldhoven · P. Buskens $(\square)$

The Netherlands Organisation for Applied Scientific

Research (TNO), De Rondom 1, 5612 AP Eindhoven, The

Netherlands

e-mail: pascal.buskens@tno.nl;

buskens@dwi.rwth-aachen.de

D. Mann $\cdot$ H. Keul $\cdot$ P. Buskens

DWI - Leibniz Institute for Interactive Materials e.V.,

Forckenbeckstrasse 50, 52056 Aachen, Germany

G. Bex

TNO Holst Centre, High Tech Campus 31,

5656 AE Eindhoven, The Netherlands
After photonic sintering, the resulting coatings are electrically conductive with an unprecedented high conductivity of $2.9 \times 10^{4} \mathrm{~S} \mathrm{~cm}^{-1}$. Furthermore, we report the first colloidal synthesis that yields CNCs with a high degree of Ag coverage on the surface, which is a prerequisite for obtaining coatings with high electrical conductivity.

Keywords Cellulose nanocrystals - TEMPO oxidation · Metallization - Dip coating · Photonic sintering $\cdot$ Sheet resistance

\section{Introduction}

An important class of advanced cellulose materials are electrically conductive composites (Shi et al. 2013).

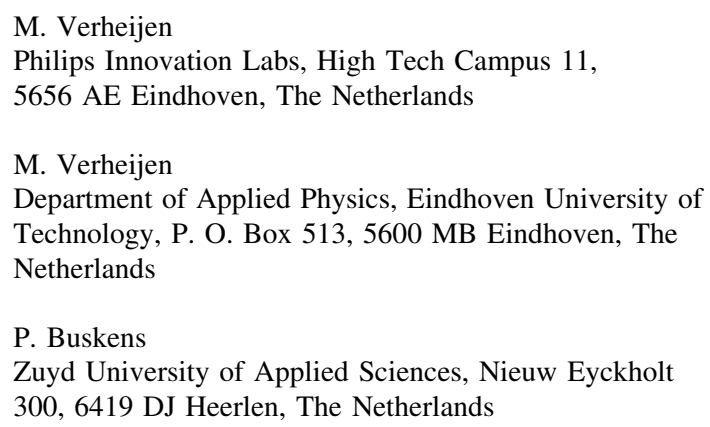


They are prepared by combining conductive materials such as polymers (Qaiser et al. 2011; Carlsson et al. 2012), metal oxides (Jur et al. 2011), metals (Zabetakis et al. 2005) and carbon (Anderson et al. 2010; Weng et al. 2011) with cellulose. The conductivity of the composites strongly depends on the conductive material applied. Using conducting polymers like polypyrrole (Carlsson et al. 2012; Raqaz et al. 2009; Shi et al. 2014), polyaniline (Qaiser et al. 2011; Xu et al. 2016) or PEDOT (Anothumakkool et al. 2015; Tkalya et al. 2013), conductivities ranging from $10^{-5}$ to $10^{2} \mathrm{~S} \mathrm{~cm}^{-1}$ were achieved (Shi et al. 2013). Alternatively, carbon nanotubes (Anderson et al. 2010) or graphene (Weng et al. 2011) were applied yielding composites with a conductivity between $10^{-5}$ and $10^{2} \mathrm{~S} \mathrm{~cm}^{-1}$ (Shi et al. 2013). The highest conductivity reported to date for cellulose composites is $10^{3} \mathrm{~S} \mathrm{~cm}^{-1}$ (Weng et al. 2011). This was achieved through use of metal oxides like $\mathrm{ZnO}$ (Jur et al. 2011) and $\mathrm{SnO}_{2}$ (Aoki et al. 2006), and metals like Ag (Zabetakis et al. 2005; Koga et al. 2014), $\mathrm{Au}$ and $\mathrm{Cu}$ (Weng et al. 2011).

Recently, our group demonstrated that silicated CNCs are excellent natural building blocks for the production of high added value, antireflective coatings (Buskens et al. 2015, 2016). The essential process steps for preparing these coatings, i.e. surface modification of CNCs dispersed in water and/or alcohol followed by application of the dispersion to glass samples using dip coating, are low cost and easily scalable. Based on this, and our previous work on $\mathrm{Ag}$ decoration of glucose-functionalized polystyrene latexes for the formation of $\mathrm{Ag}$ nanoshells (Mann et al. 2014, 2016), we hypothesized that it should be possible to decorate CNCs, which are glucose polymers, with a dense $\mathrm{Ag}$ shell through controlled reduction of $\mathrm{Ag}^{+}$, and subsequently apply the dispersion to glass substrates. Assuming that, in analogy to the silicated CNCs, the Ag modified crystals form a percolated network upon solvent evaporation, the resulting coatings should be electrically conductive. We anticipated that a high degree of coverage of the $\mathrm{CNC}$ surface with $\mathrm{Ag}$ was required for obtaining coatings with high electrical conductivity.

Here, we present a novel method for the surface decoration of needle shaped CNCs with Ag, yielding the first $\mathrm{CNC}-\mathrm{Ag}$ composite particles with a high degree of surface coverage. The resulting coatings on glass, prepared through dip coating, displayed an unprecedented high conductivity when compared to previously reported electrically conductive cellulose composites.

\section{Materials and methods}

\section{Materials}

CNC slurry (11.5 wt\% in water) was commercially obtained from the Process Development Center of the University of Maine and used without further purification. The product specification sheets are available from their website (https://umaine.edu/pdc/facilitiesavailable-for-use/nanocellulose-facility/nanocellulosespec-sheets-and-safety-data-sheets/). (2,2,6,6-Tetramethylpiperidin-1-yl)oxyl (TEMPO), $\mathrm{NaBr}, \mathrm{NaOCl}$ (11 wt $\%$ in water), $\mathrm{NaOH}, \mathrm{SnCl}_{2}, \mathrm{AgNO}_{3}$, poly(ethylene glycol) methyl ether thiol (PEG-thiol, $\mathrm{Mw}$ $\left.6000 \mathrm{~g} \mathrm{~mol}^{-1}\right), \mathrm{NH}_{3}(28-30 \mathrm{wt} \%$ in water), $\mathrm{HCl}$ and ethanol $(\mathrm{EtOH})$ were obtained from Sigma-Aldrich and used without further purification.

\section{TEMPO mediated oxidation of CNCs}

Similar to the procedure reported by Ifuku et al. (Ifuku et al. 2009), CNC slurry (90.6 g, $11.5 \mathrm{wt} \%$ in water) was diluted with demineralized (demi) water $(375 \mathrm{ml})$ and sonicated using a Branson Sonifier for $5 \mathrm{~min}$. The dispersion was added to a solution of TEMPO $(25 \mathrm{mg}$, $\left.0.16 \mathrm{mmol} \mathrm{g}^{-1} \mathrm{CNC}\right)$ and $\mathrm{NaBr}(250 \mathrm{mg})$ in demi water $(375 \mathrm{ml}) . \mathrm{NaOCl}(40 \mathrm{~g}$ of $11 \mathrm{wt} \%$ solution in water; $5.0 \mathrm{mmol} \mathrm{g}{ }^{-1} \mathrm{CNC}$ ) was added to start the oxidation, after which the $\mathrm{pH}$ of the resulting dispersion was adjusted to 10.5 through addition of $\mathrm{NaOH}$. The suspension was stirred overnight at room temperature, after which the oxidation was terminated by the addition of ethanol $(10 \mathrm{ml})$. The resulting suspension was dialyzed against demi water for 7 days until neutral $\mathrm{pH}$ was achieved in the effluent.

Isolated yield: $7.7 \mathrm{~g}$ (74\%) of oxidized CNC.

\section{Two step procedure for silver deposition on CNCs}

First step: Similar to the procedure reported by our group for $\mathrm{Ag}$ deposition on glucose-functionalized polystyrene latexes (Mann et al. 2014, 2016), a dispersion of TEMPO-oxidized CNCs $(15.0 \mathrm{~g}, 0.77$ $\mathrm{wt} \%$ in demi water) was added to a solution of $\mathrm{SnCl}_{2}$ 
(1.25 g, $5.54 \mathrm{mmol})$ and $\mathrm{HCl}(2 \mathrm{ml}, 37 \mathrm{wt} \%)$ in demi water $(100 \mathrm{ml})$. The mixture was stirred for one hour on a roller bank at room temperature, and subsequently centrifuged for $30 \mathrm{~min}$ at $10,000 \mathrm{rpm}$. Then, the supernatant was removed, and the residue dispersed in demi water $(100 \mathrm{ml})$ using an ultrasonic bath. The resulting dispersion had a solid content of $0.10 \mathrm{wt} \%$. Subsequently, the mixture was added to a solution of silver nitrate $(600 \mathrm{mg}, 3.53 \mathrm{mmol})$ and aqueous ammonia $(3.75 \mathrm{ml}, 28-30 \mathrm{wt} \%$ in water) in water $(100 \mathrm{ml})$. The resulting reaction mixture was inverted 10 times, immediately centrifuged at $10,000 \mathrm{rpm}$ for $30 \mathrm{~min}$ and dispersed in demi water $(50 \mathrm{ml})$.

Isolated yield: $0.02 \mathrm{~g} \mathrm{CNC-Ag} \mathrm{composite} \mathrm{particles}$ (45 wt $\% \mathrm{Ag}$, based on thermogravimetric analysis (TGA)).

Second step: A dispersion of CNC-Ag composite particles (16.7 ml, $0.04 \mathrm{wt} \%, 45 \mathrm{wt} \% \mathrm{Ag})$ was added to a freshly prepared Ag deposition bath. The deposition bath was prepared by combining solution 1 and 2 (Table 1) in an ice bath. Solution 2 was prepared by adding glucose and tartaric acid to a mixture of ethanol and demi water, after which the solution was placed in an ultrasonic bath for $30 \mathrm{~min}$. The particle dispersion was added to the deposition bath and stirred overnight on a roller bank. PEG-thiol (Mw 6000, $100 \mathrm{mg}$, $0.017 \mathrm{mmol}$ ) was added to stabilize the metallized CNC particles. The resulting dispersion was stirred for one hour on the roller bank, centrifuged at 10,000 rpm for $30 \mathrm{~min}$, and dispersed in demi water $(20 \mathrm{ml})$ using an ultrasonic tip ( 2 min, power level $20 \%$ ).

Isolated yield: $0.13 \mathrm{~g} \mathrm{CNC}-\mathrm{Ag}$ composite particles (94 wt\% Ag, based on TGA).

UV-VIS extinction spectrophotometry on CNCAg dispersions

For the optical characterization of aqueous CNC-Ag dispersions, we performed UV-VIS extinction spectrophotometry using a Shimadzu 3600 UV-VIS-NIR spectrophotometer. The measurements were performed in quartz cuvettes having an optical path length of $10 \mathrm{~mm}$. Spectra were recorded for the wavelength range from 300 to $700 \mathrm{~nm}$.

Thermogravimetric analysis

TGA was applied to determine the decomposition temperature of CNCs in CNC-Ag composite particles, and the weight ratio $\mathrm{CNC}$ to $\mathrm{Ag}$ using a Discovery TGA (TA Instruments). Samples were heated to $900{ }^{\circ} \mathrm{C}$ under air with a rate of $20{ }^{\circ} \mathrm{C} \mathrm{min}^{-1}$.

Coatings on float glass

Coatings comprising $\mathrm{CNC}-\mathrm{Ag}$ composite particles were applied to float glass slides from an alcoholic dispersion (1.0 wt\%) using dip coating (KSV NIMA dipcoater with control software). A withdrawal speed of $500 \mathrm{~mm} \mathrm{~min}^{-1}$ was applied. The solvent was evaporated under ambient conditions. Up to eight layers were applied on top of each other through sequential dip coating. Photonic sintering was applied to transform the dip coated nanoparticle layers into a conductive coating, using a NovaCentrix PulseForge 1300. The sample was half covered with a metal plate. The other half was exposed to light flashes for photonic sintering using diverse settings (Table 2). One sample, resulting from eight sequential dips, was cured in an oven for $3 \mathrm{~h}$ at $450{ }^{\circ} \mathrm{C}$.

Temperature monitoring during photonic sintering

To monitor the increase in coating temperature during photonic sintering, a sample with an electrical circuit containing a molybdenum line with known dimensions and temperature coefficient of resistance was covered with a thick layer of CNC-Ag particles (eight sequential dips). The line resistance was measured during photonic sintering, and directly correlated to the sintering temperature.

Table 1 Solutions for Ag deposition bath

\begin{tabular}{|c|c|c|c|}
\hline \multicolumn{2}{|c|}{ Solution 1 (in $133.6 \mathrm{ml}$ demi water) } & \multicolumn{2}{|c|}{ Solution 2 (in $66.8 \mathrm{ml} \mathrm{EtOH} / 13.4 \mathrm{ml}$ demi water) } \\
\hline $\mathrm{AgNO}_{3}$ & $\mathrm{NaOH} / \mathrm{ammonia}$ & Glucose & Tartaric acid \\
\hline $133.6 \mathrm{mg}$ & $133.6 \mathrm{mg} / 560 \mu \mathrm{l}$ & $267.2 \mathrm{mg}$ & $26.7 \mathrm{mg}$ \\
\hline
\end{tabular}


Table 2 Settings for dip coating and photonic sintering

\begin{tabular}{llccll}
\hline \# Dips & Voltage $(\mathrm{V})$ & Time $(\mu \mathrm{s})$ & \# Flashes & Frequency $(\mathrm{Hz})$ & Flash energy $\left(\mathrm{J} \mathrm{cm}^{-2}\right)$ \\
\hline 2 & 500 & 600 & 1 & & 4 \\
4 & 500 & 600 & 1 & & 4 \\
6 & 500 & 600 & 1 & & 4 \\
8 & 500 & 600 & 1 & & 4 \\
8 & 500 & 600 & 1 & & 4 \\
8 & 300 & 1000 & 10 & 1 & 1.3 \\
8 & 500 & 300 & 3 & 1 & 2 \\
\hline
\end{tabular}

\section{Zeta potential}

The zeta potential of the CNC dispersions was measured using a Malvern nano Zetasizer. All measurements were performed in translucent disposable zeta cuvettes (DTS1060C) at $25{ }^{\circ} \mathrm{C}$ with an equilibration time of $2 \mathrm{~min}$. Each measurement was repeated three times with a delay of $10 \mathrm{~s}$ in order to confirm the quality of the obtained measuring data.

\section{X-ray diffraction}

Samples are measured in Bragg-Brentano geometry with a Bruker D8 Advance diffractometer, equipped with a motorized slit with opening angle of $0.30^{\circ}$, primary and secondary soller slit of $2.5^{\circ}$ and Lynxeye detector with opening angle of $2.94^{\circ}$. Data collection is carried out at room temperature using $\mathrm{Cu} \mathrm{K \alpha}$ radiation $(\lambda=0.15406 \mathrm{~nm})$ in the $2 \theta$ region between $10^{\circ}$ and $40^{\circ}$, step size 0.02 degrees $2 \theta$. The sample is deposited on a zero background holder ( $\mathrm{Si}$ single crystal $\langle 510\rangle$ wafer) and was rotated at $15 \mathrm{rpm}$ during the measurement. Generator settings are $40 \mathrm{kV}$ and $40 \mathrm{~mA}$. Phase identification is carried out with the Bruker program "EVA4.2."

Fourier transform-infrared spectroscopy

Infrared spectroscopy was used to characterize the CNCs before and after TEMPO oxidation. Infrared spectra were recorded on a FT-IR Perkin Elmer spectrophotometer.

\section{Electron microscopy}

The dimensions and composition of CNCs and CNCAg composite particles were determined by transmission electron microscopy (TEM) using a JEOL
ARM200F operated at $80 \mathrm{kV}$. Dried dispersions of CNC typically display a high degree of aggregation as a result of drying during sample preparation. Therefore, cryogenic dispersion samples were prepared on copper-supported lacey carbon by rapid vitrification in liquid ethane.

High resolution scanning electron microscopy (SEM) images were recorded with a FEI Nova NanoSem 650 with the following settings: an acceleration voltage of $4 \mathrm{kV}$, spot size 3 , chamber vacuum of $60 \mathrm{~Pa}$ and a gaseous analytical detector, or with an acceleration voltage of $5 \mathrm{kV}$, spot size 3 , chamber vacuum of circa $1 \mathrm{mPa}$ and a through lens detector.

Titration to determine the degree of oxidation of CNCs

The carboxyl content of the CNCs after TEMPO oxidation was determined by suspending of freeze dried oxidized CNCs (50-55 mg) into $0.01 \mathrm{M}$ hydrochloric acid solution $(15 \mathrm{ml})$. The suspension was treated with an ultrasonic tip for $2 \mathrm{~min}$, after which the sample was titrated with $0.01 \mathrm{M} \mathrm{NaOH}$. The carboxyl groups content, or degree of oxidation (DO), was calculated using following equation:

$D O=\frac{162 c\left(V_{2}-V_{1}\right)}{w-36 c\left(V_{2}-V_{1}\right)}$

where $V_{1}$ and $V_{2}$ are the volumes of $\mathrm{NaOH}$ (in 1), $c$ is the concentration $\mathrm{NaOH}$ (in mol/l) and $w$ is the weight of the freeze dried sample (Habibi et al. 2006).

UV-VIS-NIR extinction spectrophotometry

Transmission of the dip coated glass samples was measured using a Shimadzu 3600 UV-VIS-NIR spectrophotometer. Spectra were recorded for the wavelength range from 300 to $1200 \mathrm{~nm}$. 
Sheet resistance

Four point probe measurements for determination of sheet resistance are not reliable on highly porous coatings on glass. The probes penetrate the coating and destroy the three-dimensional network of nanoparticles, resulting in irreproducible values. Therefore, resistances were measured between small dots of conductive silver glue, applied $8 \mathrm{~mm}$ apart from each other, on top of the CNC-Ag coatings. The sheet resistance $R_{S}$ can be calculated as described by Wolf and Tauber (1986).

$R_{S}=\frac{W\left(R_{T}-2 R_{C}\right)}{L}$

with $W$ as distance between the dots of silver glue (i.e. $8 \mathrm{~mm}), R_{T}$ as total measured resistance and $R_{C}$ as contact resistance. The conductivity is calculated from the sheet resistance $R_{S}$ of the coating and its layer thickness $t$

$\sigma=\frac{1}{R_{S} t}$

\section{Layer thickness}

Layer thickness measurements were performed using a Dektak XT profilometer from Bruker, with $1 \mathrm{mg}$ stylus force. The coating thickness was determined on five different positions for each coated sample. Average values are presented in this report.

\section{Results and discussion}

\section{CNC-Ag composite particles}

The aim of our study was to prepare CNC-Ag composite particles with a high degree of $\mathrm{Ag}$ coverage on the surface of the CNCs, and to use these Agdecorated CNCs for the preparation of electrically conductive coatings. Very recently, our group reported a two-step procedure for the deposition of $\mathrm{Ag}$ on glucose-functionalized polystyrene particles, yielding polystyrene-Ag core-shell particles with a high degree of Ag coverage on the latex surface ( $>90 \%$ ) (Mann et al. 2016). We selected this metallization procedure for Ag deposition on CNCs (Fig. 1). At the start of the reported procedure for metallization of the latex particles, $\mathrm{Sn}^{2+}$ ions were coordinated to the surface of glucose-functionalized polystyrene particles to serve as nucleation points for the reductive deposition of $\mathrm{Ag}$ from $\mathrm{Ag}^{+}$(Mann et al. 2014, 2016). This pretreatment step is commonly used to deposit $\mathrm{Ag}$ on macroscopic surfaces, and has first been applied as pretreatment in a multistep process for deposition of Ag on spherical colloids by Liz-Marzán and coworkers (Kobayashi et al. 2001).

They demonstrated that $\mathrm{Sn}^{2+}$ ions coordinated to the surface of spherical silica particles and reduced $\mathrm{Ag}^{+}$to $\mathrm{Ag}$, while $\mathrm{Sn}^{2+}$ oxidized to $\mathrm{Sn}^{4+}$. Coordination of $\mathrm{Sn}^{2+}$ ions to the polystyrene particle surface was demonstrated by the strong increase in zeta potential (Mann et al. 2014, 2016). However, after pretreatment of $\mathrm{CNCs}_{\text {with }} \mathrm{SnCl}_{2}$ and subsequent removal of excess salt through centrifugation and washing, we noticed that the zeta potential remained unchanged at $-42.1 \mathrm{mV}$. Ergo, the $\mathrm{Sn}^{2+}$ ions did not coordinate to the CNC surface.

Because it is well known that $\mathrm{Sn}^{2+}$ coordinates to carboxylate groups, we decided to use surface oxidized CNCs as templates for reductive Ag deposition. Following the procedure developed by Ifuku et al. (Ifuku et al. 2009), we subjected the CNCs to TEMPO mediated oxidation (Saito and Isogai 2004). The use of this technique has attracted a number of investigations, which demonstrated that only the hydroxymethyl groups of polysaccharides were oxidized, whereas the secondary hydroxyls remained unaffected (Ifuku et al. 2009). After TEMPO oxidation and subsequent purification of the CNCs, we analyzed the surfaceoxidized CNCs using cryo-TEM (Fig. 2a), XRD (Fig. 2b) and FT-IR spectrometry (Fig. 2c), and compared their properties to those of the non-oxidized crystals. As demonstrated by the cryo-TEM (Fig. 2a) and XRD analyses (Fig. 2b) of untreated and oxidized CNCs, the oxidation did not significantly affect the $\mathrm{CNC}$ size and crystallinity, respectively. The average length and width of both the untreated and oxidized needle-shaped CNCs is 150-200 and $5 \mathrm{~nm}$, respectively. Both materials show the $\mathrm{X}$-ray fingerprint which is typical for crystalline cellulose (Buskens et al. 2015). In the FT-IR spectrum, the presence of carboxylic acid groups on the surface of oxidized $\mathrm{CNCs}$ is confirmed by the occurrence of the absorption band at $1730 \mathrm{~cm}^{-1}$ representing the $\mathrm{C}=\mathrm{O}$ stretching vibration (Fig. 2c, Online Resource 1a) (Li et al. 2015). 
Fig. 1 Schematic representation of TEMPO oxidation of CNCs, and subsequent two-step metallization procedure for Ag deposition on oxidized $\mathrm{CNCs}$
TEMPO oxidation of CNCs:

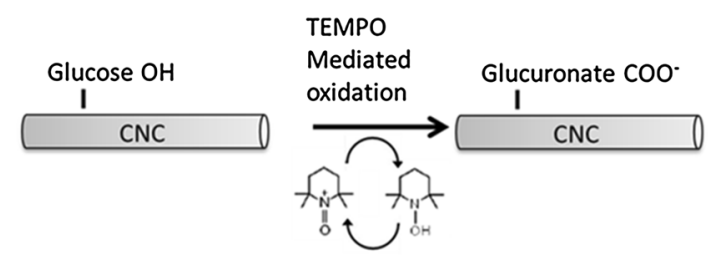

Two-step metallization procedure for Ag deposition on CNCs:

Glucuronate $\mathrm{COO}^{\circ}$

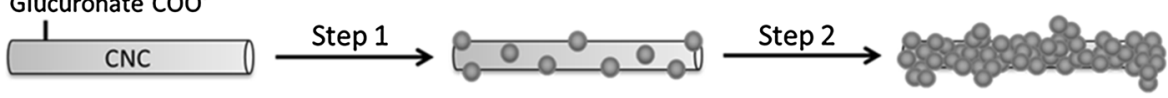

Furthermore, we noticed an additional absorption band at $1601 \mathrm{~cm}^{-1}$ for the TEMPO oxidized CNCs, representing the $\mathrm{COO}-$ antisymmetric stretching in carboxylic acid salts. From the FT-IR spectrum of TEMPO oxidized CNCs, we calculated a degree of oxidation (DO) of 0.110 (Habibi et al. 2006). Furthermore, we noticed from the FT-IR spectra that neither the total crystallinity index $(T C I=1.00$ and $\left.T C I_{o x}=0.99\right)$ nor the lateral order index $(L O I=1.00$ and $L O I_{o x}=1.01$ ) changed upon TEMPO oxidation (Li et al. 2015). The hydrogen bond intensity increased upon TEMPO oxidation from $H B I=0.06$ to $H B I_{o x}=$ 0.11 (Li et al. 2015), which can be expected upon formation of carboxylic acid groups.

For a more accurate determination of the DO, we performed a conductometric acid-base titration using the method reported by Vignon and coworkers (Fig. 3) (Da Silva Perez et al. 2003; Habibi et al. 2006).

Based on these results, we obtained a carboxyl

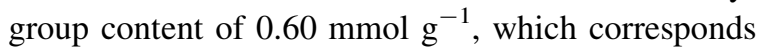
to a $D O$ of 0.105 . This is in good agreement with the $D O$ obtained through FT-IR analysis.

The stability of the dispersions of unmodified and oxidized CNCs at neutral $\mathrm{pH}$ was determined through measurement of the zeta potential. For the unmodified and oxidized CNCs, we determined a zeta potential of -42.1 and $-43.5 \mathrm{mV}$, respectively, which indicates that both dispersions are stable at neutral $\mathrm{pH}$.

To validate the surface coordination of $\mathrm{Sn}^{2+}$ ions, the dispersion of oxidized CNCs was mixed with an aqueous solution of $\mathrm{SnCl}_{2}$. After one hour at room temperature, excess $\mathrm{SnCl}_{2}$ was removed through centrifugation and washing, and the resulting CNCs were dispersed in demi water. After treatment, we measured a significant increase in zeta potential from -43.5 to $+2.14 \mathrm{mV}$, demonstrating successful coordination of $\mathrm{Sn}^{2+}$ ions to the carboxylate groups of the oxidized CNCs. This was confirmed by TEMEDX analysis of the TEMPO oxidized CNCs after treatment with $\mathrm{SnCl}_{2}$ (Fig. 4).

In the first metallization step, the $\mathrm{Sn}^{2+}$ modified $\mathrm{CNCs}$ were subjected to an aqueous solution of $\mathrm{AgNO}_{3}$ and $\mathrm{NH}_{3}$ at room temperature. An immediate colour change due to the formation of $\mathrm{Ag}$ nanoparticles on CNCs, with their distinct localized plasmon resonance at about $420 \mathrm{~nm}$, indicated that $\mathrm{Ag}$ was formed rather quickly (Fig. 5a). The reaction mixture was inverted 10 times at room temperature, after which the Ag-modified CNCs were isolated through centrifugation. The resulting supernatant was transparent in the visible, indicating that no free $\mathrm{Ag}$ particles were formed, and that the metallization procedure is highly selective for CNC surface decoration. The Ag-modified CNCs were then washed and dispersed in demi water. The Ag content was determined using TGA (Fig. 5b). The UV-Vis spectrum displays a rather broad extinction peak with its maximum at a wavelength of $420 \mathrm{~nm}$, which represents the localized plasmon resonance of the $\mathrm{Ag}$ nanoparticles deposited on CNCs (Fig. 5a). In the TGA, the Ag-decorated CNCs display approximately $5 \%$ mass loss due to dehydration, followed by a distinct temperature profile for degradation with degradation temperatures of 190 and $350{ }^{\circ} \mathrm{C}$ (Fig. 5b). The $\mathrm{Ag}$ residue obtained after full decomposition of cellulose at a temperature above $550{ }^{\circ} \mathrm{C}$ represents 45 $\mathrm{wt} \%$ of the original CNC-Ag composite particles.

Morphology and architecture of the CNC-Ag composite particles, obtained after the first metallization step, were analyzed using high resolution cryoTEM (Fig. 6). The cryo-TEM image displays a bundle of maximum $5 \mathrm{CNCs}$ inhomogeneously decorated 
Fig. 2 Characterization of TEMPO oxidized CNCs in comparison to untreated CNCs; a cryo-TEM images, b X-ray diffractograms (y axis are arbitrary units), and c FT-IR spectra

a
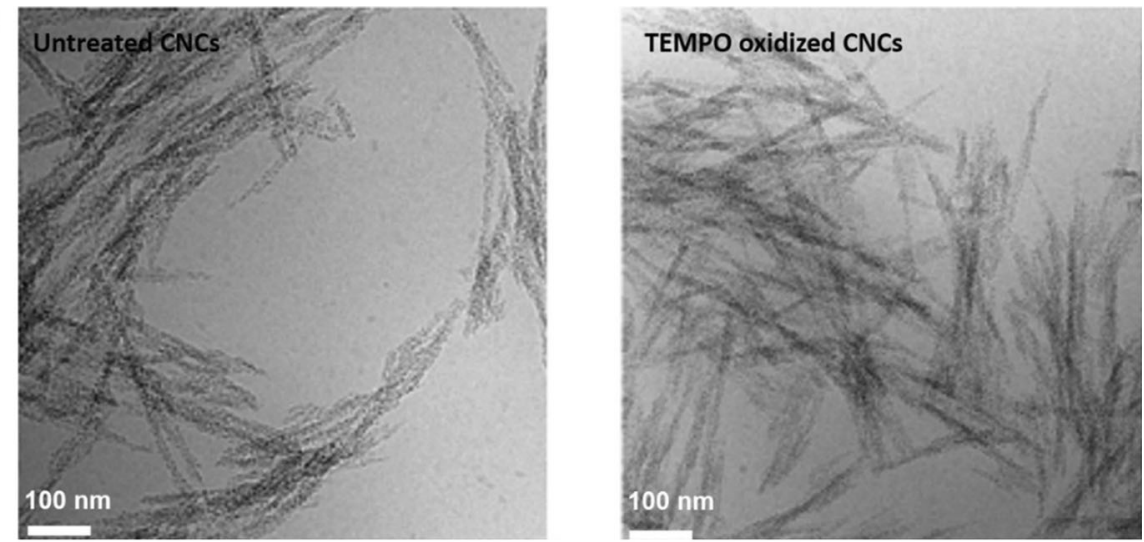

b

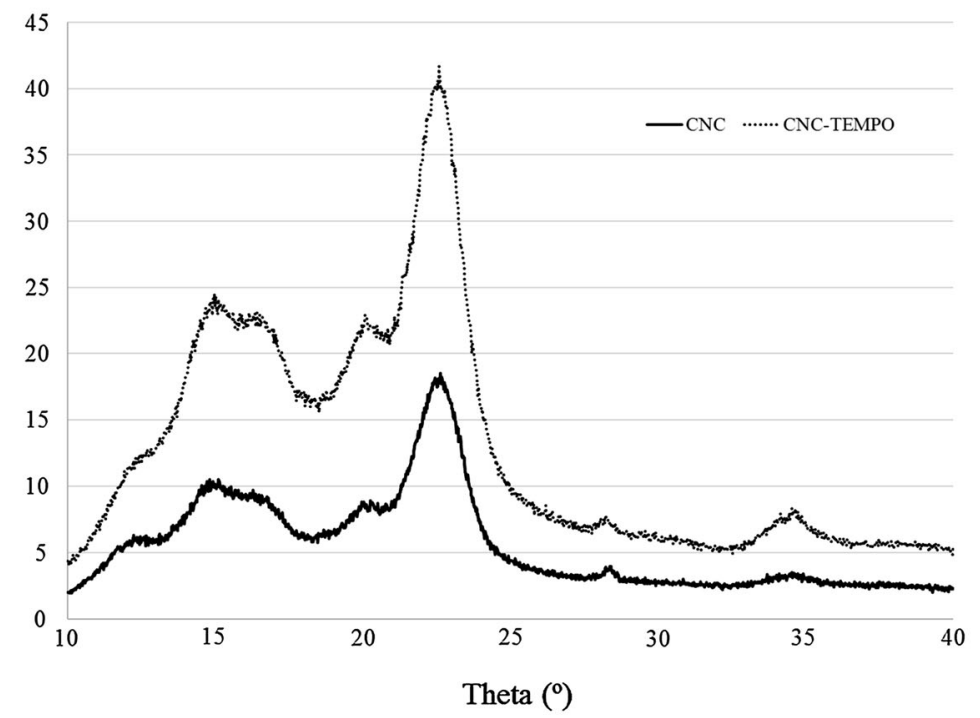

c

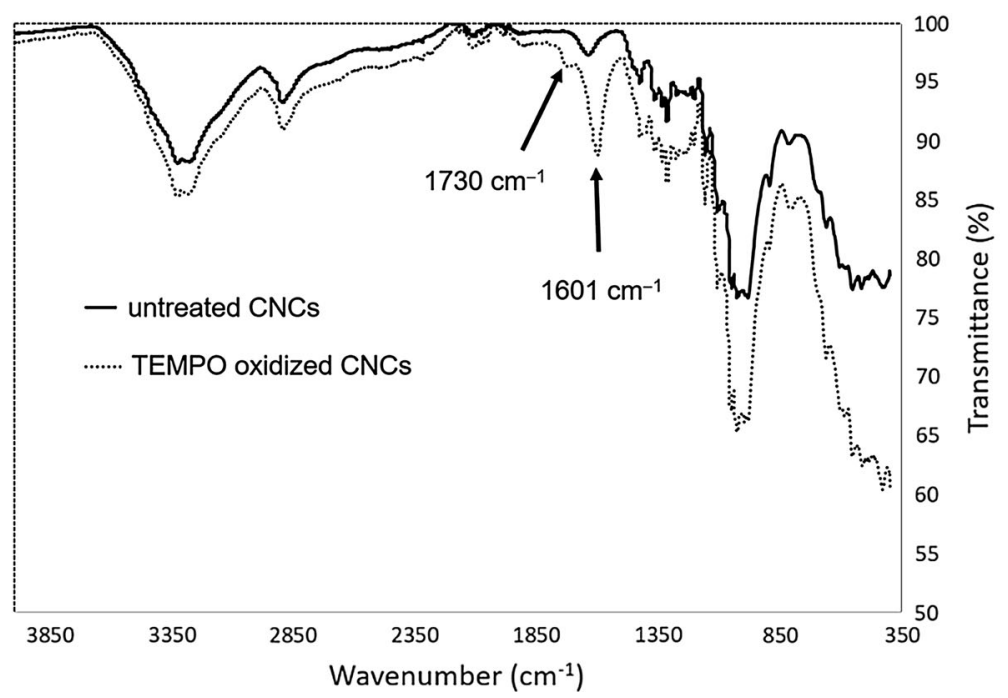


Fig. 3 Titration curve of the conductometric acidbase titration of TEMPO oxidized CNCs (amount of CNCs $w=0.0495 \mathrm{~g}$; concentration $\mathrm{NaOH}$ $c=0.0110 \mathrm{~mol} \mathrm{1}^{-1}$ )
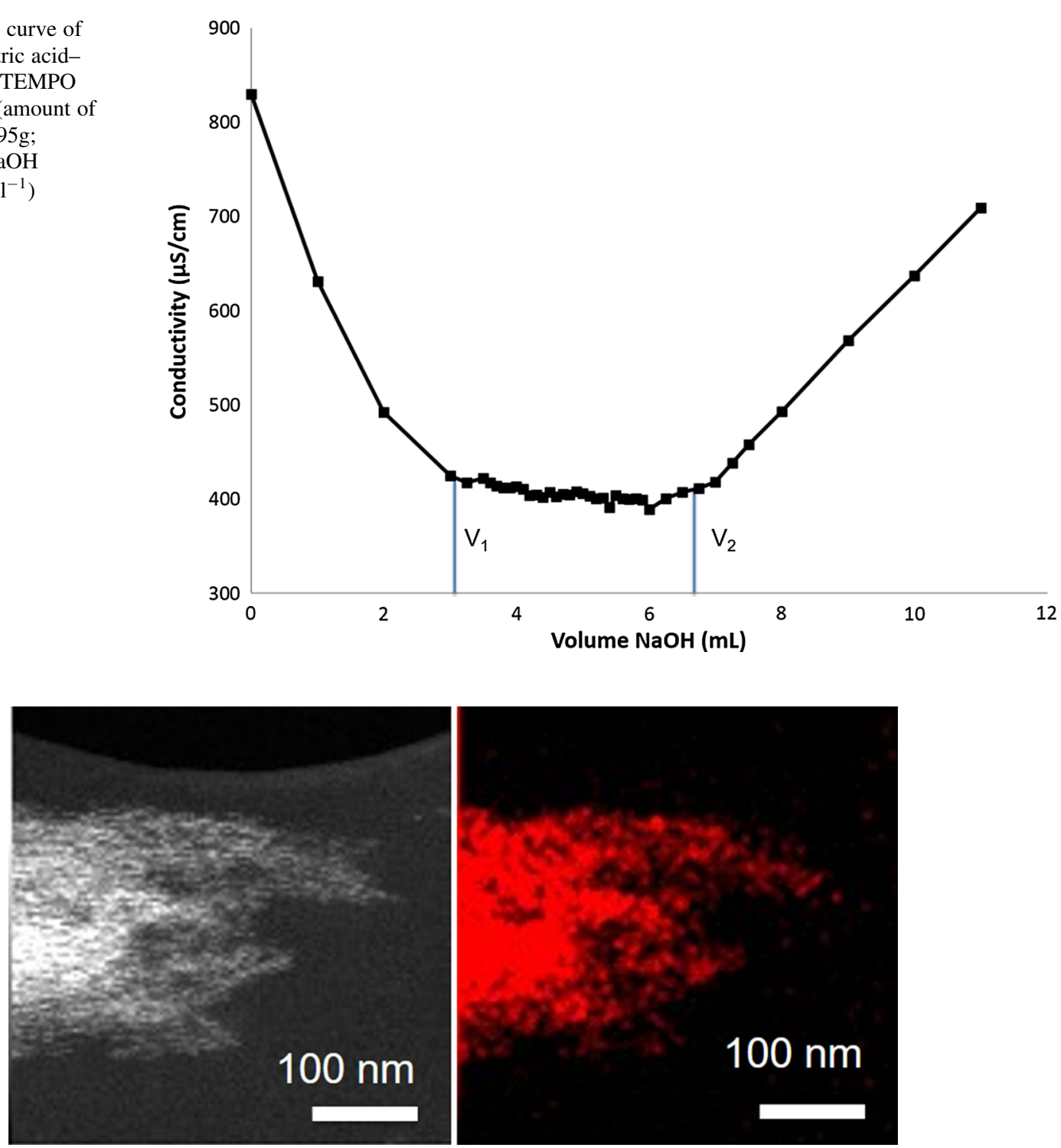

Fig. 4 TEM of the TEMPO oxidized CNCs after treatment with $\mathrm{SnCl}_{2}$ and TEM-EDX analysis for Sn (red). (Color figure online)

with individual spherical Ag nanoparticles of a size between 5 and $10 \mathrm{~nm}$ (pointed out by the red arrow in Fig. 6). This matches the UV-VIS extinction peak at $420 \mathrm{~nm}$.

In analogy to the procedure reported by Buskens and co-workers for Ag deposition on glucose-modified polystyrene latex particles (Mann et al. 2016), we applied a second step for further deposition and growth of Ag nanoparticles. In this step, the CNC-Ag composite particles were subjected to a $\mathrm{Ag}$ deposition bath at $0{ }^{\circ} \mathrm{C}$. This bath was prepared through combination of two individually prepared solutions: an aqueous solution of
$\mathrm{Ag}\left[\mathrm{NH}_{3}\right]_{2}^{+}$complex and a solution of glucose and tartaric acid in a mixture of ethanol and demi water. After finishing the Ag deposition, PEG-SH was added to ensure colloidal stability of the CNC-Ag composite particles. The particles were purified through centrifugation and washing, and dispersed in demi water. Similarly to the first process step, the supernatant was transparent indicating that $\mathrm{Ag}$ was selectively deposited on the CNC surface. TEM analysis of the resulting CNC-Ag composite particles revealed an unprecedented high level of surface coverage with Ag particles of a size up to about $50 \mathrm{~nm}$ (Fig. 7). 
Fig. 5 a UV-VIS spectrum of Ag-modified CNCs dispersed in demi water and b TGA of Ag-modified $\mathrm{CNCs}$; both after the first metallization step
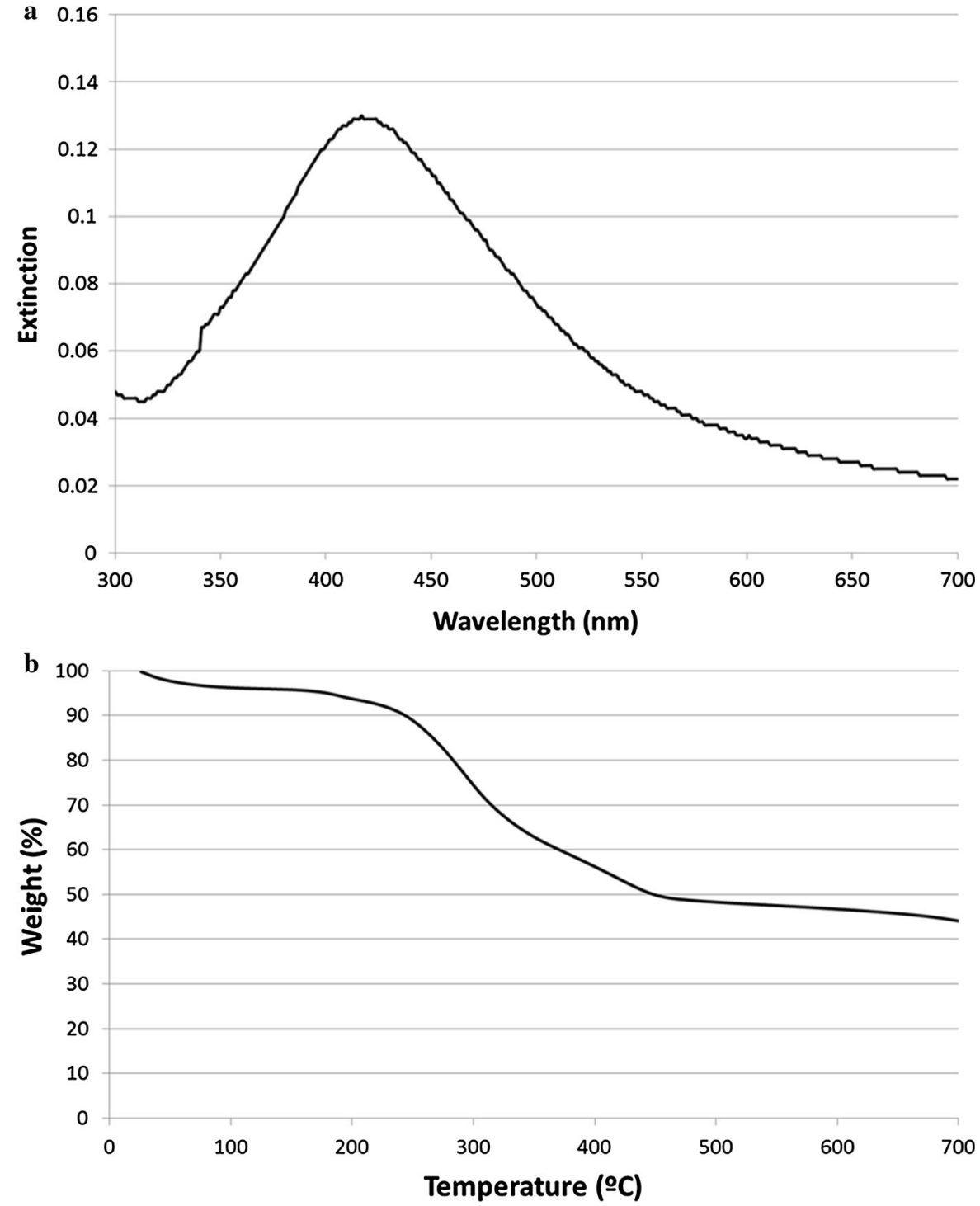

This unprecedented high level of surface coverage was achieved both through the formation of new $\mathrm{Ag}$ particles on the CNC surface and through the growth of previously existing $\mathrm{Ag}$ nanoparticles. It is well known that metal nanoparticles such as $\mathrm{Ag}$ and $\mathrm{Pd}$ deposited on the surface of a dielectric particle, act as a catalyst for further $\mathrm{Ag}$ deposition and thereby ensure that reduction of further $\mathrm{Ag}^{+}$ions only takes place on the nanoparticle surface (Kobayashi et al. 2001). TGA analysis showed that the amount of $\mathrm{Ag}$ in the composite particles increased during the second process step from 45 to $94 \mathrm{wt} \%$ (Online Resource 1b). UV-VIS spectrophotometry yielded an extinction spectrum (Online Resource 1c) almost identical to the one for the CNC-Ag composites after the first process step (Fig. 5a).

\section{Conductive coatings}

For the preparation of conductive coatings comprising the CNC-Ag composite particles obtained after the second process step, we prepared a $1.0 \mathrm{wt} \%$ dispersion in ethanol. The particles were applied to float glass slides through dip coating using the particle dispersion as coating liquid. We used a withdrawal speed of $500 \mathrm{~mm} \mathrm{~min}^{-1}$, and applied multiple layers of 


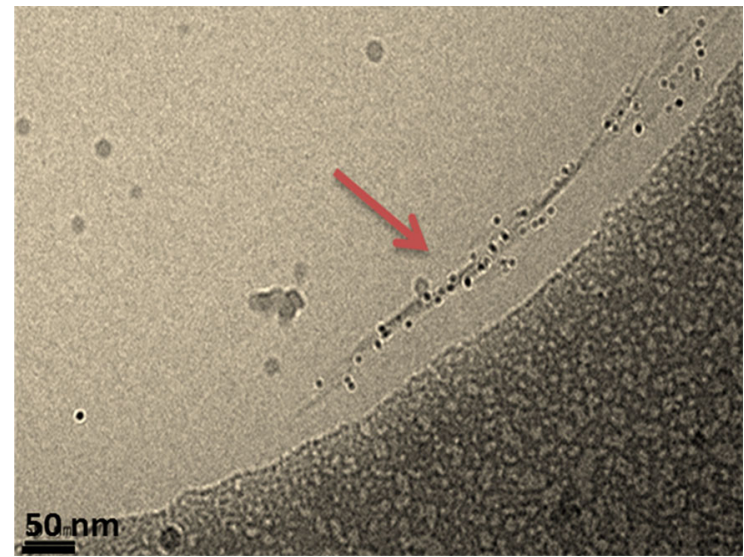

Fig. 6 Cryo-TEM image of CNC-Ag composite particles obtained after the first metallization step

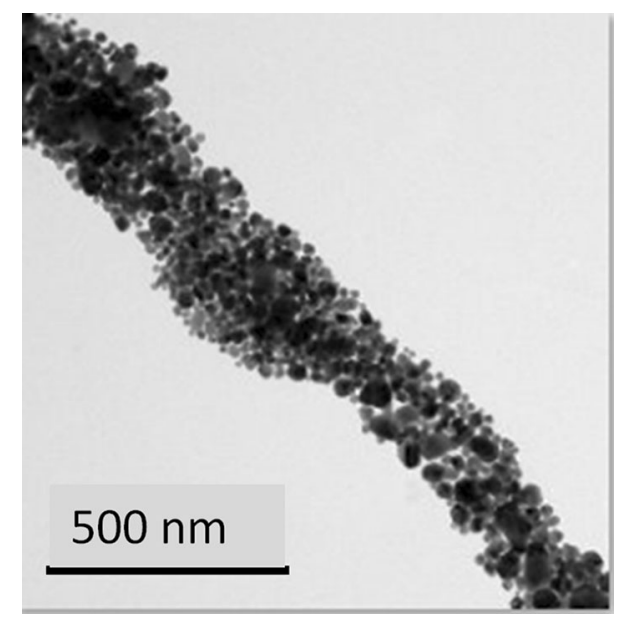

Fig. 7 TEM analysis of CNC-Ag composites formed after the second process step

particles on top of each other through sequential dipping and drying. After evaporation of all volatiles, we photonically sintered the thin film using pulsed light from a flash lamp. This is a common technique for sintering of metal inks, and is frequently used in printed electronics (Schroder 2011). Sintered samples resulting from 2, 4, 6 and 8 sequential dips were analyzed using SEM (Fig. 8). As depicted in the SEM images, the degree of surface coverage and the coating layer thickness $t$ increase with increasing number of dips. For the sample resulting from 8 sequential dips, we performed SEM analysis before photonic sintering (Fig. 9a). From comparison between the sintered (Fig. 8d) and non-sintered coating, we conclude that the morphology of the structures did not significantly change during photonic sintering. Furthermore, we performed a reference experiment through application of an oven cure (Fig. 9b).

By applying a coating (8 sequential dips) on top of an electrical circuit containing a molybdenum line with known dimensions and temperature coefficient of resistance, we determined that the maximum temperature reached during photonic sintering was approximately $450{ }^{\circ} \mathrm{C}$ (Online Resource 1d). Therefore, we performed the oven cure at that temperature for a duration of $3 \mathrm{~h}$.

After removal of the coating from one side of the substrate, we determined the UV-Vis transmission using spectrophotometry (Fig. 10). The average transmission in the visible $(425-675 \mathrm{~nm}) T_{v i s}$ decreased with increasing layer thickness from $92.7 \%$ (uncoated float glass) to $18.0 \%$ (4 dips), $12.7 \%$ (6 dips) and $5.9 \%$ (8 dips) (Fig. 10, Table 3, Online Resource 1e). The morphology change of the oven cured samples is reflected in the transmission curve yielding an average visible transmission of $28.6 \%$. Using a Dektak profilometer, we determined the average layer thickness $t$ of the coatings obtained after 4, 6, and 8 dips (Table 3). As expected, the average layer thickness increased with increasing number of sequential dips from $70.5 \mathrm{~nm}$ (4 dips) to $92.6 \mathrm{~nm}$ (6 dips) and $99.4 \mathrm{~nm}$ (8 dips). For flat and dense coating layers of the same material applied on top of each other in sequential dips, the coating thickness should linearly increase with the number of dips, provided that the same liquid coating formulation and withdrawal speed are used. Apparently, this is not the case for the porous coatings consisting of CNC-Ag nanoparticles. With increasing number of dips, the average coating thickness achieved per dip decreases $(17.6 \mathrm{~nm}$ for 4 dips, $15.4 \mathrm{~nm}$ for 6 dips, $12.4 \mathrm{~nm}$ for 8 dips). Nonetheless, it is reasonable to expect that the same amount of coating material is applied during each dip, which means that with increasing thickness of the porous layers, the additional coating material is preferably deposited inside the porous network instead of on top.

Regular probes used in four point probe measurements for the determination of the sheet resistance of the coating penetrate the particle layer, and consequently destroy the three dimensional coating network. Therefore, this method is not suited for determining the conductivity. Instead, we measured resistances between 
a

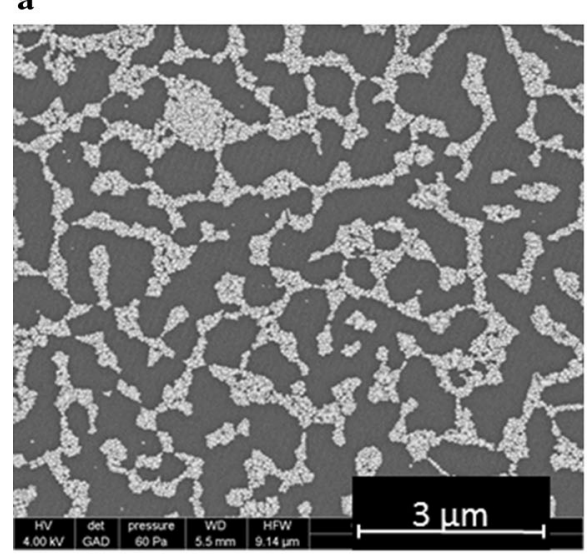

c

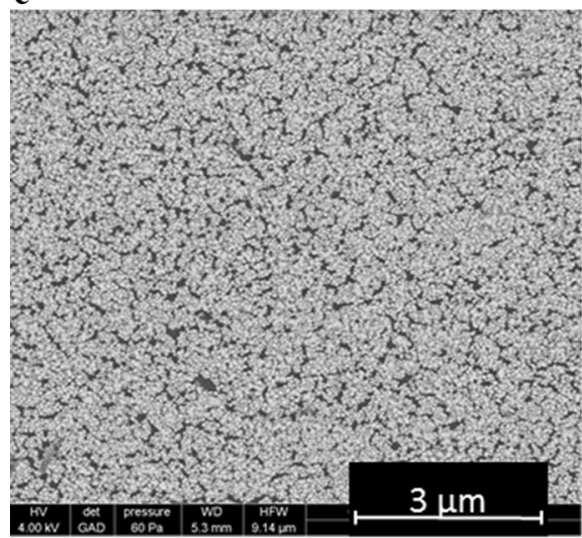

b

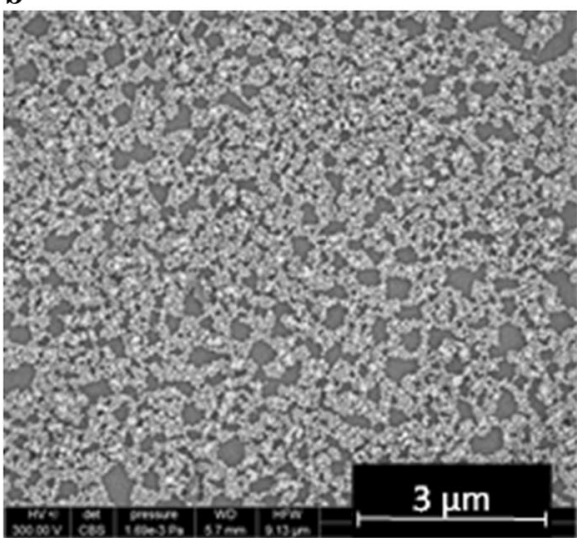

d

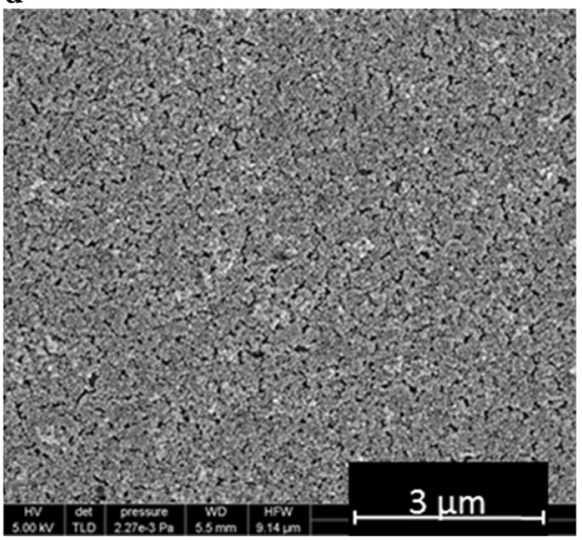

Fig. 8 Coated samples resulting from a 2, b 4, c 6 and d 8 sequential dips after photonic sintering

$\mathbf{a}$

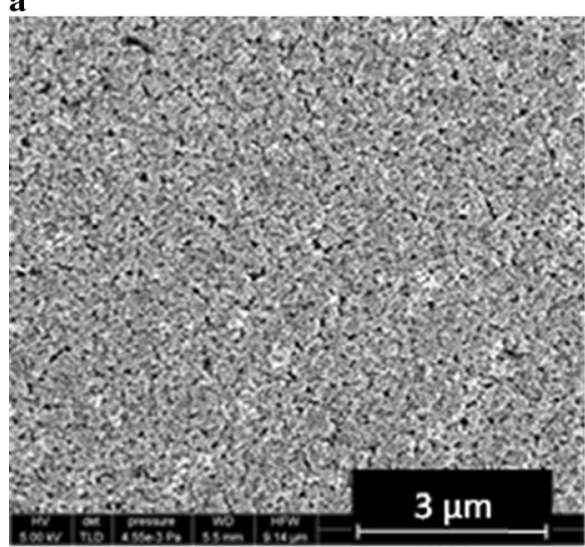

b

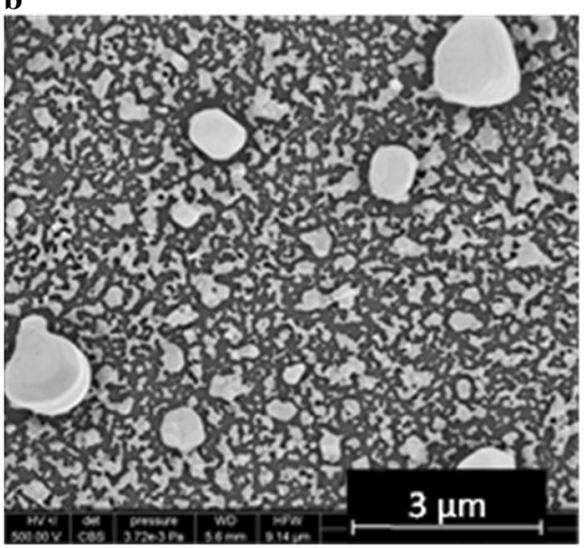

Fig. 9 Coating from 8 sequential dips a before photonic sintering, and b after $3 \mathrm{~h}$ oven cure at $450{ }^{\circ} \mathrm{C}$

small dots of conductive silver glue applied at distances of $8,16,24$ and $32 \mathrm{~mm}$ from each other. When plotting the measured resistance $R_{T}$ between these contact points as function of their distance $L$ (Fig. 11), sheet $R_{S}$ and contact resistance $R_{C}$ can be derived from the slope and axis intersection, respectively, 
Fig. 10 Transmission of glass samples single-sided coated with $\mathrm{CNC}-\mathrm{Ag}$ composite particles

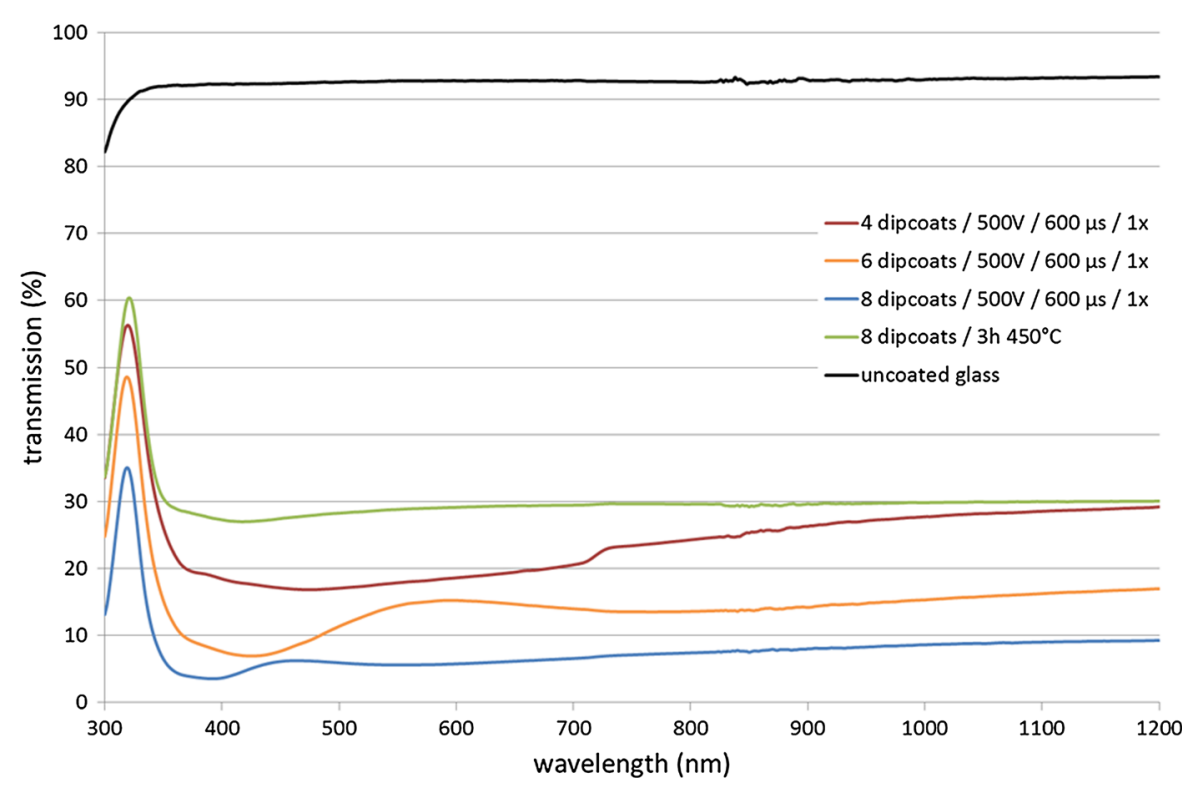

Table 3 Thickness, visible transmission and conductivity of photonically sintered coatings (settings photonic sintering: $U=500 \mathrm{~V}, 1$ flash, flash time $=600 \mu \mathrm{s}, E=4.0 \mathrm{~J} \mathrm{~cm}^{-2}$ )

\begin{tabular}{llcl}
\hline \# Dips & $t(\mathrm{~nm})$ & $T_{\text {vis }}(\%)$ & $\sigma\left(\mathrm{S} \mathrm{cm}^{-1}\right)$ \\
\hline 4 & 70.5 & 18.0 & $5.4 \times 10^{-1}$ \\
6 & 92.6 & 12.7 & $3.5 \times 10^{3}$ \\
8 & 99.4 & 5.9 & $2.9 \times 10^{4}$ \\
\hline
\end{tabular}

$R_{T}=\frac{R_{S}}{W} L+2 R_{C}$

with $W$ as width of the conducting path (Wolf and Tauber 1986).

For the coatings prepared using 6 and 8 sequential dips, the sheet resistances were determined using 10 (Online Resource 1f) and $20 \mathrm{~mm}$ wide conducting paths (Fig. 11). The average sheet resistance values resulting from these measurements are $31 \Omega \mathrm{sq}^{-1}(6$ dips, $t=92.6 \mathrm{~nm})$ and $3.6 \Omega \mathrm{sq}^{-1}(8 \mathrm{dips}$, $t=99.4 \mathrm{~nm})$. The corresponding linear regressions display a coefficient of determination $R^{2}$ of 0.99 , which underlines that the regression line excellently fits the experimental data points. This is not the case for the sample resulting from 4 sequential dips: using a linear regression, the sheet resistance of this sample could be determined as $5.6 \times 10^{5} \Omega \mathrm{sq}^{-1}$ (4 dips, $t=70.5 \mathrm{~nm}$ ), albeit with a poor fit of the regression line with the experimental data points $\left(R^{2}=0.80\right.$ 0.86 , Online Resource $1 \mathrm{~g}$ ). Note that the decrease in sheet resistance cannot simply be explained by an increase in coating thickness with increasing number of dips. Although the difference in coating thickness between the layers produced in 6 and 8 sequential dips is only minor, the difference in the number of conductive $\mathrm{CNC}-\mathrm{Ag}$ nanoparticles per surface area of substrate is more than $30 \%$ (vide supra). The conductivity $\sigma$ of these coatings can be derived from $R_{S}$ and layer thickness $t$,

$\sigma=\frac{1}{R_{S} t}$

and was calculated as $5.4 \times 10^{-1} \mathrm{~S} \mathrm{~cm}^{-1}$, $3.5 \times 10^{3} \mathrm{~S} \mathrm{~cm}^{-1}$, and $2.9 \times 10^{4} \mathrm{~S} \mathrm{~cm}^{-1}$, respectively. Latter conductivity exceeds the conductivity of the best performing cellulose composites reported to date by a factor $\sim 30$ (Shi et al. 2013).

\section{Conclusions}

We have successfully developed a conductive coating consisting of Ag-decorated CNCs, with a conductivity that exceeds the best performing cellulose composites reported to date by a factor 30 . In line with our previous work on CNC-based antireflective coatings, we demonstrated that surface modified $\mathrm{CNCs}$ are interesting building blocks for application in high tech functional coatings, and realized the first conductive coating based on CNCs. Here, we decorated the CNC surface with $\mathrm{Ag}$ 
Fig. 11 a Schematic representation of the measured samples and $\mathbf{b} R_{T}$ as a function of $L$ using conducting paths of $20 \mathrm{~mm}$ width (squares data points for coating resulting from 6 sequential dips, triangles data points for coating resulting from 8 sequential dips) a
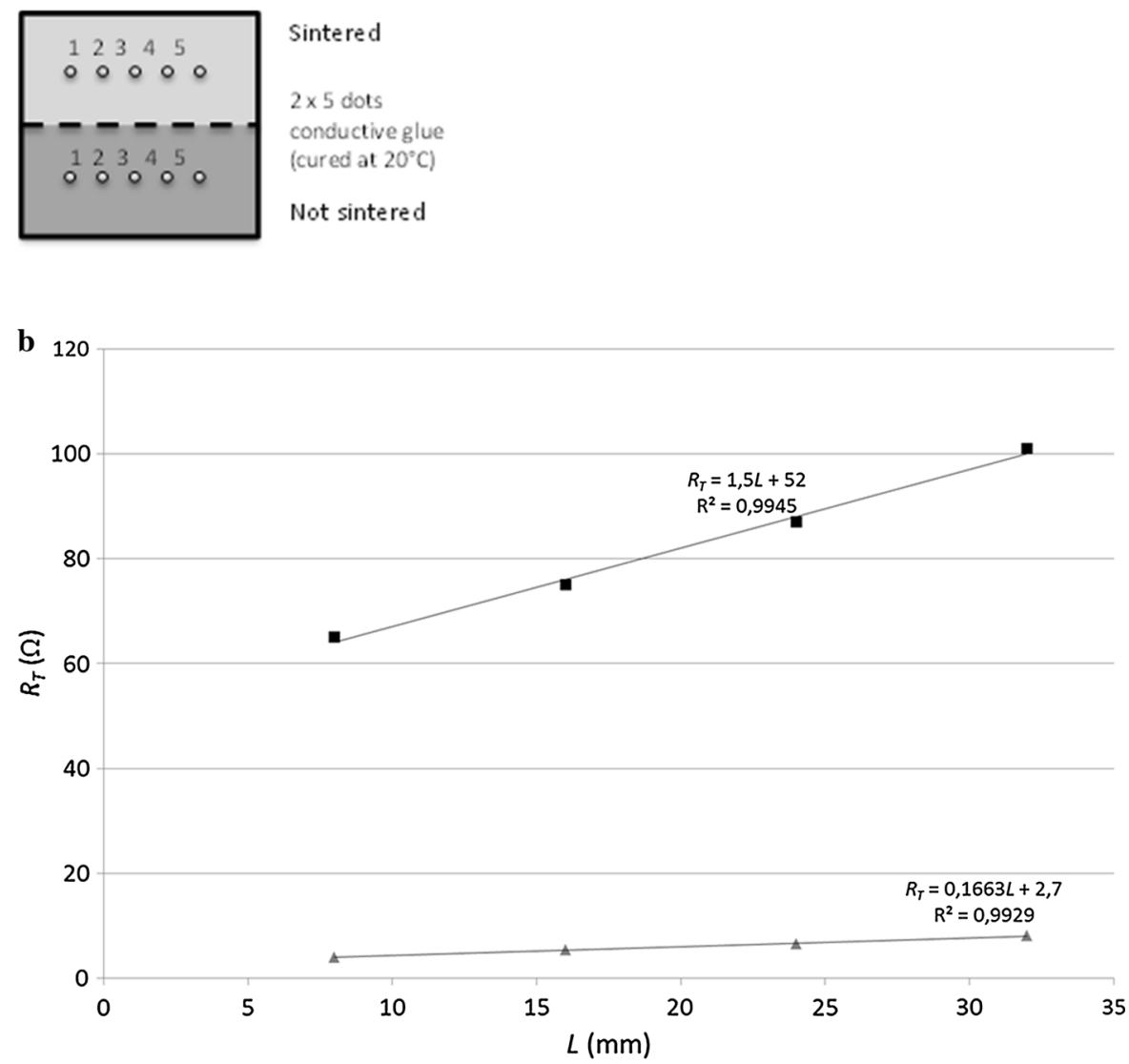

using a two-step metallization procedure, and realized CNC-Ag composite particles with an unprecedented high degree of metal coverage. These were dispersed in ethanol and applied to glass substrates through dip coating. The resulting particle layers were photonically sintered, yielding coatings with an electrical conductivity up to $2.9 \times 10^{4} \mathrm{~S} \mathrm{~cm}^{-1}$.

Acknowledgments The authors affiliated to TNO gratefully acknowledge the European Commission for funding this work as part of the EU Project GREENANOFILMS. This project has received funding from the European Union Seventh Framework Program (FP7/2007-2013) under Grant Agreement No. 603519. Solliance is acknowledged for funding the high resolution TEM facility.

Open Access This article is distributed under the terms of the Creative Commons Attribution 4.0 International License (http:// creativecommons.org/licenses/by/4.0/), which permits unrestricted use, distribution, and reproduction in any medium, provided you give appropriate credit to the original author(s) and the source, provide a link to the Creative Commons license, and indicate if changes were made.

\section{References}

Anderson RE, Guan J, Ricard M, Dubey G, Su J, Lopinski G, Dorris G, Bourne O, Simard B (2010) Multifunctional single-walled carbon nanotube-cellulose composite paper. J Mater Chem 20:2400-2407

Anothumakkool B, Soni R, Bhange SN, Kurungot S (2015) Novel scalable synthesis of highly conducting and robust PEDOT paper for a high performance flexible solid supercapacitor. Energy Environ Sci 8:1339-1347

Aoki Y, Huang J, Kunitake T (2006) Electro-conductive nanotubular sheet of indium tin oxide as fabricated from the cellulose template. J Mater Chem 16:292-297

Buskens P, Mourad M, Meulendijks N, Van Ee R, Burghoorn M, Verheijen M, Van Veldhoven E (2015) Highly porous, ultra-low refractive index coatings produced through random packing of silicated cellulose nanocrystals. Coll Surf A $487: 1-8$

Buskens P, Burghoorn M, Mourad MCD, Vroon Z (2016) Antireflective coatings for glass and transparent polymers. Langmuir 32:6781-6793

Carlsson DO, Nyström G, Zhou Q, Berglund LA, Nyholm L, Strømme M (2012) Electroactive nanofibrillated cellulose aerogel composites with tunable structural and electrochemical properties. J Mater Chem 22:19014-19024 
Da Silva Perez D, Montanari S, Vignon MR (2003) TEMPOmediated oxidation of cellulose III. Biomacromolecules 4:1417-1425

Habibi Y, Chanzy H, Vignon MR (2006) TEMPO-mediated surface oxidation of cellulose whiskers. Cellulose 13:679-687

Ifuku S, Tsuji M, Morimoto M, Saimoto H, Yano H (2009) Synthesis of silver nanoparticles templated by TEMPOmediated oxidized bacterial cellulose nanofibers. Biomacromolecules 10:2714-2717

Jur JS, Sweet WJ III, Oldham CJ, Parsons GN (2011) Atomic layer deposition of conductive coatings on cotton, paper and synthetic fibers: conductivity analysis and functional chemical sensing using "all-fiber" capacitors. Adv Funct Mater 21:1993-2002

Kobayashi Y, Salgueriño-Maceira V, Liz-Marzán LM (2001) Deposition of silver nanoparticles on silica spheres by pretreatment steps in electroless plating. Chem Mater 13:1630-1633

Koga H, Nogi M, Komoda N, Nge TT, Sugahara T, Suganuma K (2014) Uniformly connected conductive networks on cellulose nanofiber paper for transparent paper electronics. NPG Asia Mater 6:e93

Li B, Xu W, Kronlund D, Määttänen A, Liu J, Smätt J-H, Peltonen J, Willför S, Mu X, Xu C (2015) Cellulose nanocrystals prepared via formic acid hydrolysis followed by TEMPO-mediated oxidation. Carbohydr Polym 133:605-612

Mann D, Chattopadhyay S, Pargen S, Verheijen M, Keul H, Buskens P, Möller M (2014) Glucose-functionalized polystyrene particles designed for selective deposition of silver on the surface. RSC Adv 4:62878-62881

Mann D, Nascimento-Duplat D, Keul H, Möller M, Verheijen M, Xu M, Urbach PH, Adam AJL, Buskens P (2016) The influence of particle size distribution and shell imperfections on the plasmon resonance of $\mathrm{Au}$ and $\mathrm{Ag}$ nanoshells. Plasmonics. doi:10.1007/s11468-016-0345-8

Qaiser AA, Hyland MM, Patterson DA (2011) Surface and charge transport characterization of polyaniline-cellulose acetate composite membranes. J Phys Chem B 115:1652-1661

Raqaz A, Mihranyan A, Welch K, Nyholm L, Strømme M (2009) Influence of the type of oxidant on anion exchange properties of fibrous cladophora cellulose/polypyrrole composites. J Phys Chem B 113:426-433

Saito T, Isogai A (2004) TEMPO-mediated oxidation of native cellulose. The effect of oxidation conditions on chemical and crystal structures of the water-insoluble fractions. Biomacromolecules 5:1983-1989

Schroder KA (2011) Mechanisms of photonic curing ${ }^{\text {TM. }}$ : processing high temperature films on low temperature substrates. Nanotech 2:220-223 (CRC Press: Boca Raton, FL)

Shi Z, Phillips GO, Yang G (2013) Nanocellulose electroconductive composites. Nanoscale 5:3194-3201

Shi Z, Gao H, Feng J, Ding B, Cao X, Kuga S, Wang Y, Zhang L, Cai J (2014) In situ synthesis of robust conductive cellulose/polypyrrole composite aerogels and their potential application in nerve regeneration. Angew Chem Int Ed 53:5380-5384

Tkalya E, Ghislandi M, Thielemans W, van der Schoot P, de With G, Koning C (2013) Cellulose nanowhiskers templating in conductive polymer nanocomposites reduces electrical percolation threshold 5-fold. ACS Macro Lett 2:157-163

Weng Z, Su Y, Wang D-W, Li F, Du J, Cheng H-M (2011) Graphene-cellulose paper flexible supercapacitors. Adv Energy Mater 1:917-922

Wolf S, Tauber RN (1986) Silicon processing for the VLSI era, volume 1: process technology. Lattice Press, Sunset Beach

Xu D, Fan L, Gao L, Xiong Y, Wang Y, Ye Q, Yu A, Dai H, Yin Y, Cai J, Zhang L (2016) Micro-nanostructured polyaniline assembled in cellulose matrix via interfacial polymerization for applications in nerve regeneration. ACS Appl Mater Interfaces 8:17090-17097

Zabetakis D, Dinderman M, Schoen P (2005) Metal-coated cellulose fibers for use in composites applicable to microwave technology. Adv Mater 17:734-738 